\title{
Global distribution and environmental suitability for chikungunya virus, 1952 to 2015
}

EO Nsoesie 1234 , MU Kraemer ${ }^{25}$, N Golding ${ }^{67}$, DM Pigott ${ }^{46}$, 0J Brady ${ }^{6}$, CL Moyes ${ }^{6}$, MA Johansson ${ }^{89}$, PW Gething ${ }^{10}$, R

Velayudhan ${ }^{11}$, K Khan ${ }^{12}$, SI Hay ${ }^{4613}$, JS Brownstein ${ }^{131314}$

1. Children's Hospital Informatics Program, Boston Children's Hospital, Boston, United States

2. These authors contributed equally to this manuscript as first authors

3. Department of Pediatrics, Harvard Medical School, Boston, United States

4. Institute of Health Metrics and Evaluation, University of Washington, Seattle, United States

5. Spatial Ecology and Epidemiology Group, Department of Zoology, University of Oxford, United Kingdom

6. Oxford Big Data Institute, Li Ka Shing Centre for Health Information and Discovery, University of Oxford, United Kingdom

7. Department of BioScience, University of Melbourne, Australia

8. Centers for Disease Control and Prevention, San Juan, Puerto Rico

9. Center for Communicable Disease Dynamics, Harvard T. H. Chan School of Public Health, Boston, United States

10. Malaria Atlas Project, Oxford Big Data Institute, Li Ka Shing Centre for Health Information and Discovery, University of Oxford, United Kingdom

11. World Health Organization, Geneva, Switzerland

12. Li Ka Shing Knowledge Institute, Division of Infectious Diseases, St Michael's Hospital, Toronto, Canada

13. These authors contributed equally to the manuscript as last authors

14. Department of Epidemiology, Biostatistics and Occupational Health, McGill University, Montreal, Canada

Correspondence: Elaine O. Nsoesie (en22@uw.edu), Moritz U.G. Kraemer (moritz.kraemer@zoo.ox.ac.uk)

Citation style for this article:

Nsoesie EO, Kraemer MU, Golding N, Pigott DM, Brady OJ, Moyes CL, Johansson MA, Gething PW, Velayudhan R, Khan K, Hay SI, Brownstein JS. Global

distribution and environmental suitability for chikungunya virus, 1952 to 2015. Euro Surveill. 2016;21(20):pii=30234. DOI: http://dx.doi.org/10.2807/1560-7917.

ES.2016.21.20.30234

Chikungunya fever is an acute febrile illness caused by the chikungunya virus (CHIKV), which is transmitted to humans by Aedes mosquitoes. Although chikungunya fever is rarely fatal, patients can experience debilitating symptoms that last from months to years. Here we comprehensively assess the global distribution of chikungunya and produce high-resolution maps, using an established modelling framework that combines a comprehensive occurrence database with bespoke environmental correlates, including up-to-date Aedes distribution maps. This enables estimation of the current total population-at-risk of CHIKV transmission and identification of areas where the virus may spread to in the future. We identified 94 countries with good evidence for current CHIKV presence and a set of countries in the New and Old World with potential for future CHIKV establishment, demonstrated by high environmental suitability for transmission and in some cases previous sporadic reports. Aedes aegypti presence was identified as one of the major contributing factors to CHIKV transmission but significant geographical heterogeneity exists. We estimated 1.3 billion people are living in areas at-risk of CHIKV transmission. These maps provide a baseline for identifying areas where prevention and control efforts should be prioritised and can be used to guide estimation of the global burden of CHIKV.

\section{Introduction}

Recent emergence and re-emergence of chikungunya virus (CHIKV) in several regions globally underscores the importance of implementing and strengthening surveillance systems for rapid and accurate case identification [1]. CHIKV causes an acute febrile illness with severe arthralgia [2]. It is a mosquito-borne pathogen of the genus Alphavirus and transmitted to and between humans by Aedes mosquitoes with four circulating genotypes [3,4]. In the eastern part of Africa CHIKV is sustained in an enzootic, sylvatic cycle that involve arboreal mosquito vectors and non-human primates [5]. Clinical manifestations of the disease usually include fever, rash and arthralgia, which are similar to dengue symptoms, potentially leading to misdiagnosis and underreporting in the absence of specific laboratory diagnostic testing [6]. CHIKV and dengue also share the same primary vector Ae. aegypti and occasional co-infection has been reported [7]. Although it has been shown that also Ae. albopictus can transmit the virus between humans such outbreaks have been more localised [8]. Mortality from CHIKV infection is rare, infected persons can experience prolonged joint pain and arthritis over a period of months to years. There are also no antiviral agents or treatments for chikungunya fever (CHIK), but candidate vaccines are under development [9].

In 2013, the first autochthonous transmission of CHIKV in the western hemisphere was reported on Saint 


\section{FIGURE 1}

Environmental covariates used in this study to predict the global environmental suitability of chikungunya virus transmission

A.

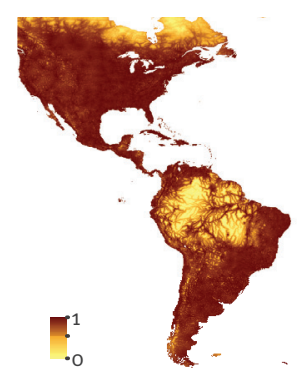

C.

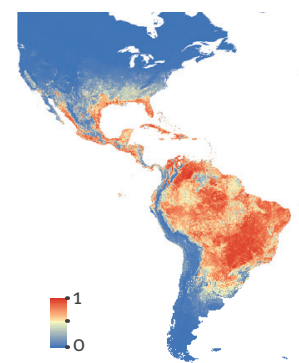

E.

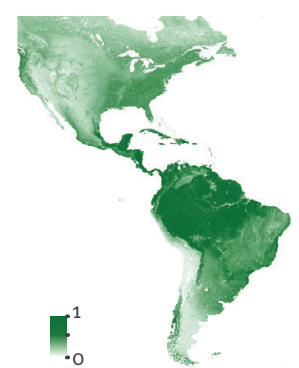

G.

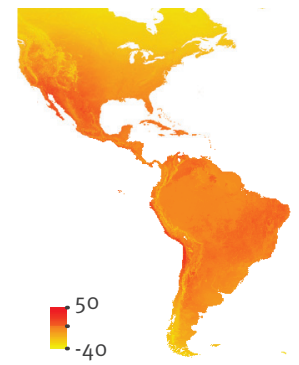

B.
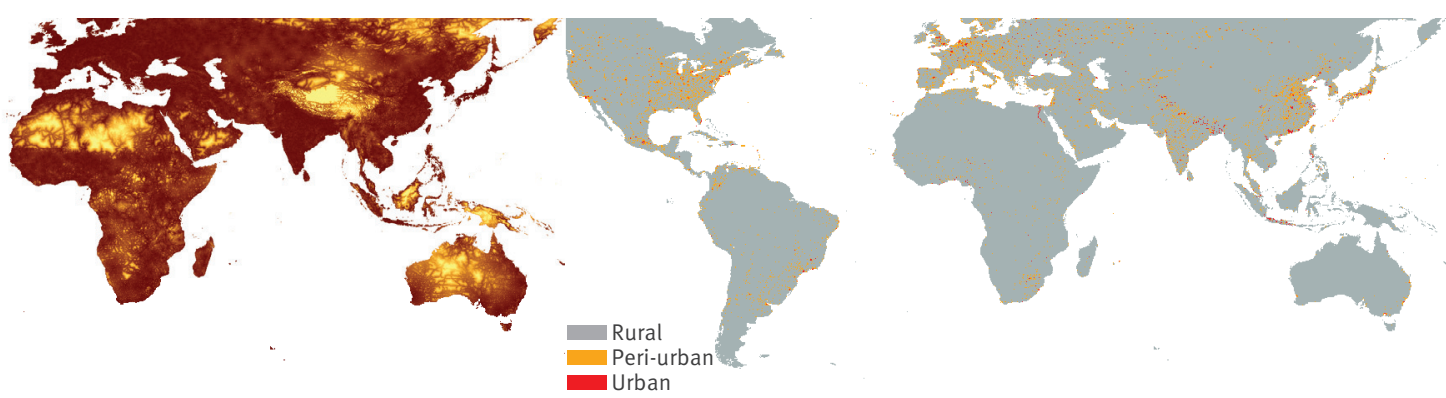

D.
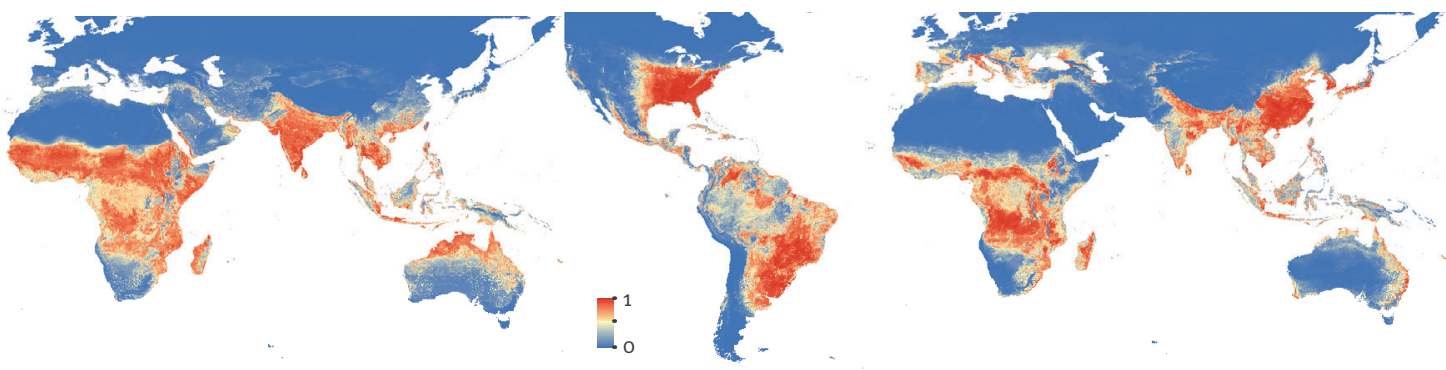

F.
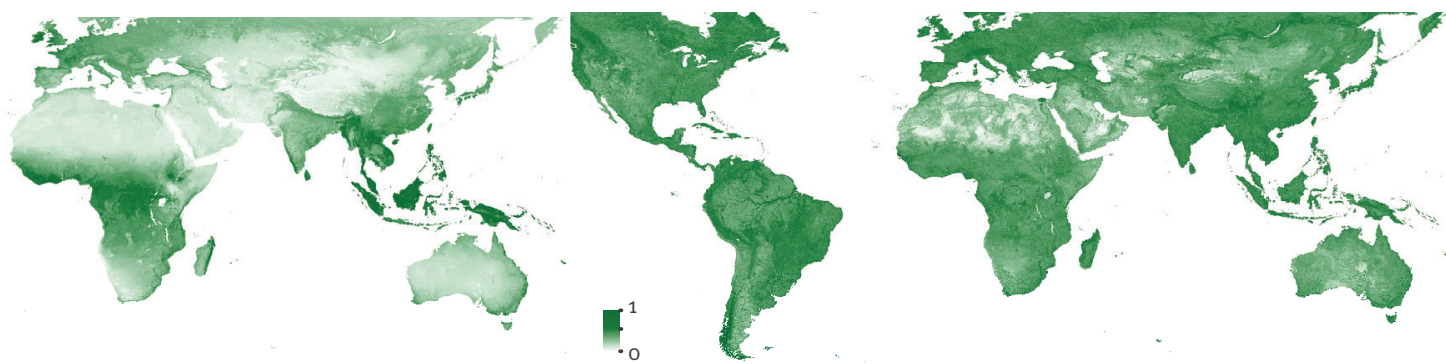

H.

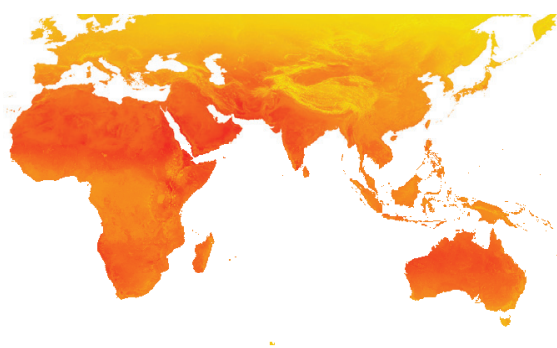

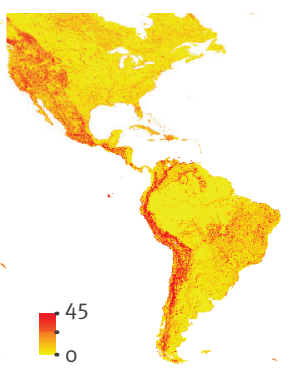

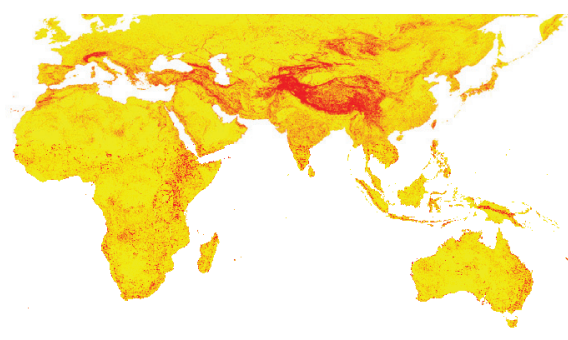

EVI: enhanced vegetation index; LST: land surface temperature.

A. Urban accessibility, with brown representing high relative accessibility to urban areas and yellow representing rural isolated areas; B. Urban, peri-urban and rural areas; C. Aedes aegypti suitability, with red representing high environmental suitability for the vector and blue representing low suitability; D. Ae. albopictus suitability, with red representing high environmental suitability for the vector and blue representing low suitability; E. EVI mean values, whereby dark green represents areas with year round vegetation growth and light green represents areas with low moisture levels; F. EVI range; G. LST mean values, whereby orange represents high temperatures and yellow lower relative temperatures; H. LST range, with orange representing high variation throughout an average year and yellow representing little annual variation. 


\section{FIGURE 2}

Distribution per continent of chikungunya virus transmission occurrence points from peer-reviewed evidence and health organisations, 1952-May 2015

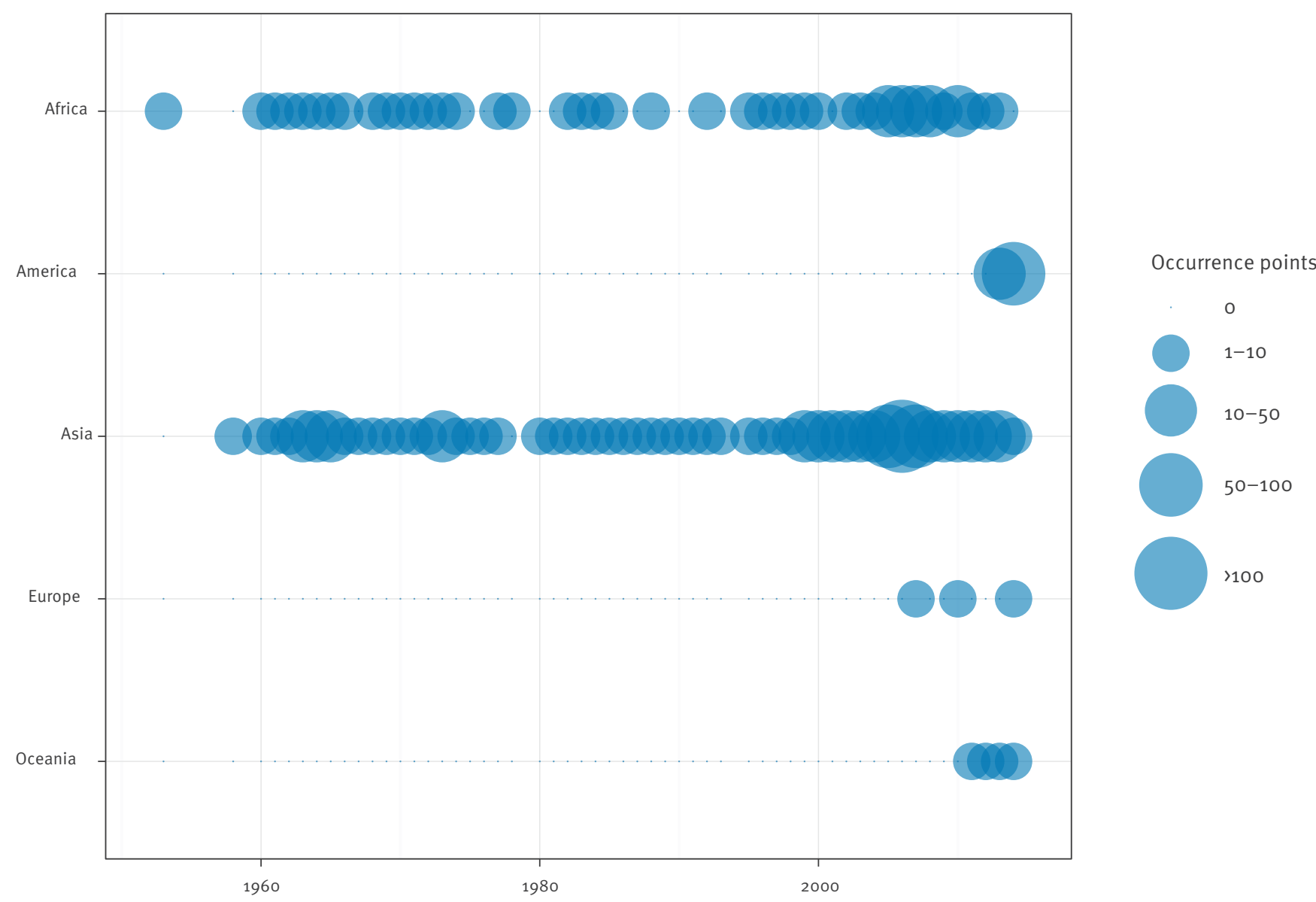

Collection year

Martin Island in the Caribbean. The outbreak subsequently spread to several Caribbean islands and the wider Americas (including many countries in Central and northern South America), reaching over one million suspected and confirmed cases by December 2014 [10] due to the presence of a large naïve population and competent vectors. Despite an increasing global spatial distribution and clinical significance, detailed spatial information of the global geographical extent of CHIKV remains sparse. Previous work has mostly focused on assessment of CHIKV presence at a country level [1]. A comprehensive assessment of CHIKV transmission records globally is critical to identify knowledge gaps and regions where preventive and targeted control efforts should be prioritised. Additionally, this information can be used to guide estimation of the global clinical burden of CHIKV, which is currently unknown. Studies on the prediction of future trends and possibility of transmission in unaffected regions would also benefit from a thorough evaluation of the contemporary distribution and mapping of the extent of CHIKV on a fine spatial scale.
To assess the global distribution of CHIKV, we adopt established disease mapping approaches [11] previously used for dengue [12,13], Zika [14], and the leishmaniases [15]. We developed a comprehensive database of unique locations where CHIKV transmission has been reported and an evidence consensus score - a summary statistic of evidence for CHIKV presence or absence using a weighted scoring system [13]. We then couple these data with a comprehensive set of environmental correlates and contemporary Aedes distribution maps in a species distribution model to generate high-resolution risk maps $(5 \mathrm{~km} \times 5 \mathrm{~km})$, representing environmental suitability for CHIKV transmission and to derive population-at-risk estimates. These maps and estimates can be refined as new data on disease and vector range expansion become available.

\section{Methods}

\section{Occurrence records}

Data on CHIKV transmission events were obtained from peer-reviewed literature, health organisations, and supplementary evidence such as, data on vector presence. A comprehensive review of peer-reviewed 
Evidence consensus (a) and predicted environmental suitability (b) for chikungunya virus in Africa and Europe, as of May 2015
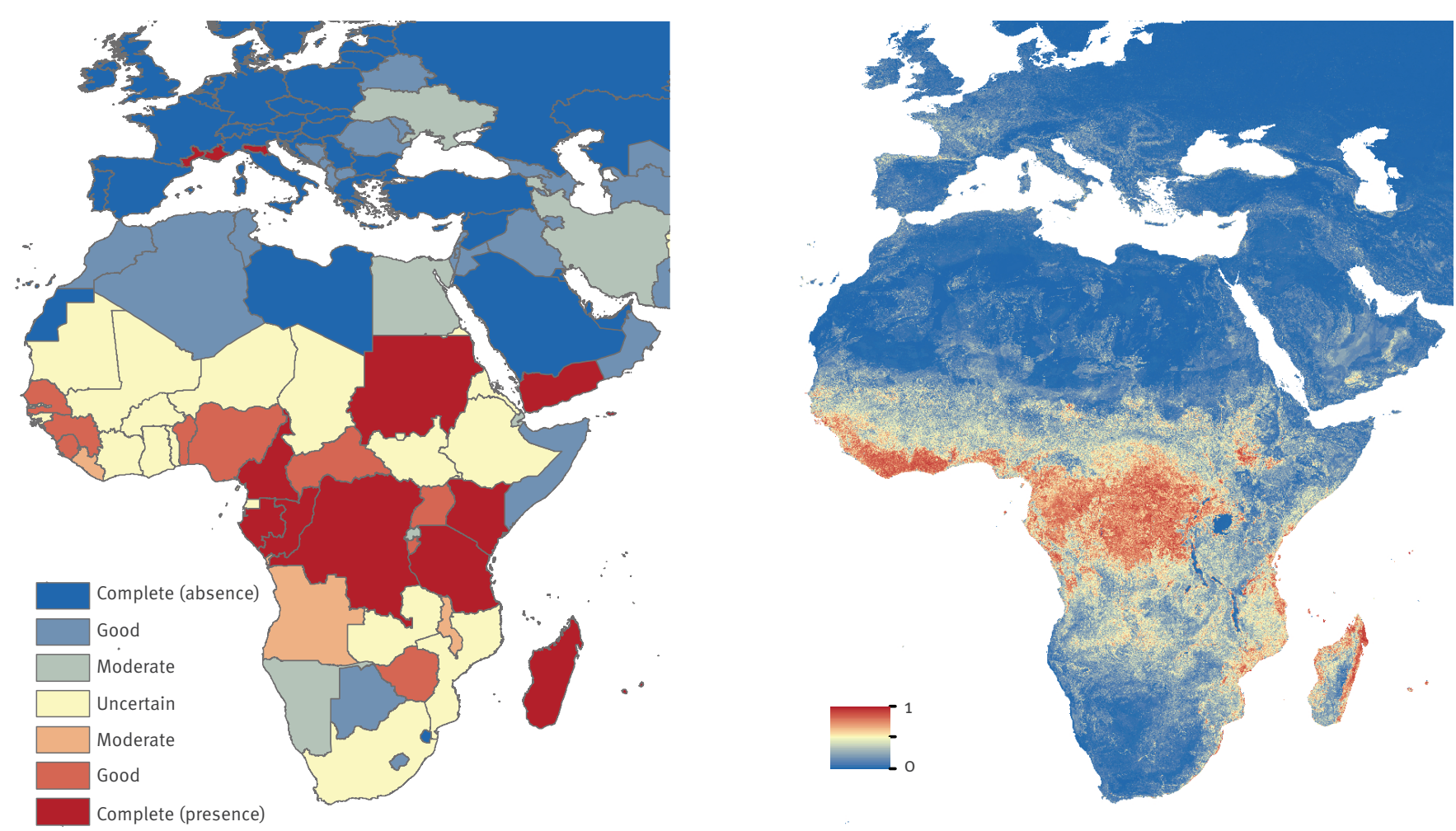

The colour legend for the evidence consensus ranges from red to blue, representing complete consensus on presence to complete consensus on absence. Indeterminate consensus is in yellow. Evidence consensus is presented at Admin1 level for Italy and France, and Admino level for all other countries. The predicted environmental suitability map is presented in similar colours with 1 and o representing most and least suitability for chikungunya virus, respectively.

literature was initially conducted on 4 April 2014 in PubMed using the search term 'chikungunya'. The data were subsequently updated every month until 7 May 2015. A total of 2,120 articles were identified. Abstracts of all articles were read to identify those that possibly included geographical information of clinical cases, epidemiological records or outbreaks of CHIKV. Information was extracted on: (i) date of occurrence, (ii) type of event (autochthonous vs imported cases), (iii) number of cases, and (iv) the geographical location (latitude, longitude and administrative level) following a standard protocol [16]. If people were diagnosed after returning from travel but the location of infection could be identified unambiguously due to information on the travel history, the data were retained. Of the 2,120 articles, 339 were used, resulting in 463 unique occurrence points.

We also consulted health organisation webpages, including the World Health Organization (WHO), Pan American Health Organization (PAHO), Global Infectious Disease and Epidemiology Network (GIDEON) and the United States (US) Centers for Disease Control and Prevention (CDC). Data on recent and ongoing outbreaks in the Americas were obtained from weekly PAHO epidemiological reports. Since PAHO is a WHO affiliate organisation, PAHO and WHO data were combined to represent a single health organisation data source resulting in 65 unique occurrence points.

Finally, 600 occurrence points based on reports of CHIKV outbreaks were obtained from the online surveillance system, HealthMap (http://www.healthmap. org/en).

Each location was then classified as precise (e.g. a town) or an administrative unit and the latter were linked to the appropriate polygon information from the Global Administrative Unit Layers dataset (http:// www.gadm.org). Each occurrence point was attributed to a specific location (latitude and longitude) based on the level of detail presented in the data source. We used Google Maps (https://www.google.co.uk/ maps) to support the geopositioning. The database subsequently underwent temporal and spatial standardisation using a standard protocol described elsewhere [16]. We removed any duplicate records so that the resulting database contained only one occurrence record per spatial entity. Further, any records reported from the above mentioned data sources that fell outside land borders (e.g. due to wrong geopositioning of the original source) were removed. Occurrence records 


\section{FIGURE 4}

Evidence consensus (a) and predicted environmental suitability (b) for chikungunya virus in the Americas, as of May 2015
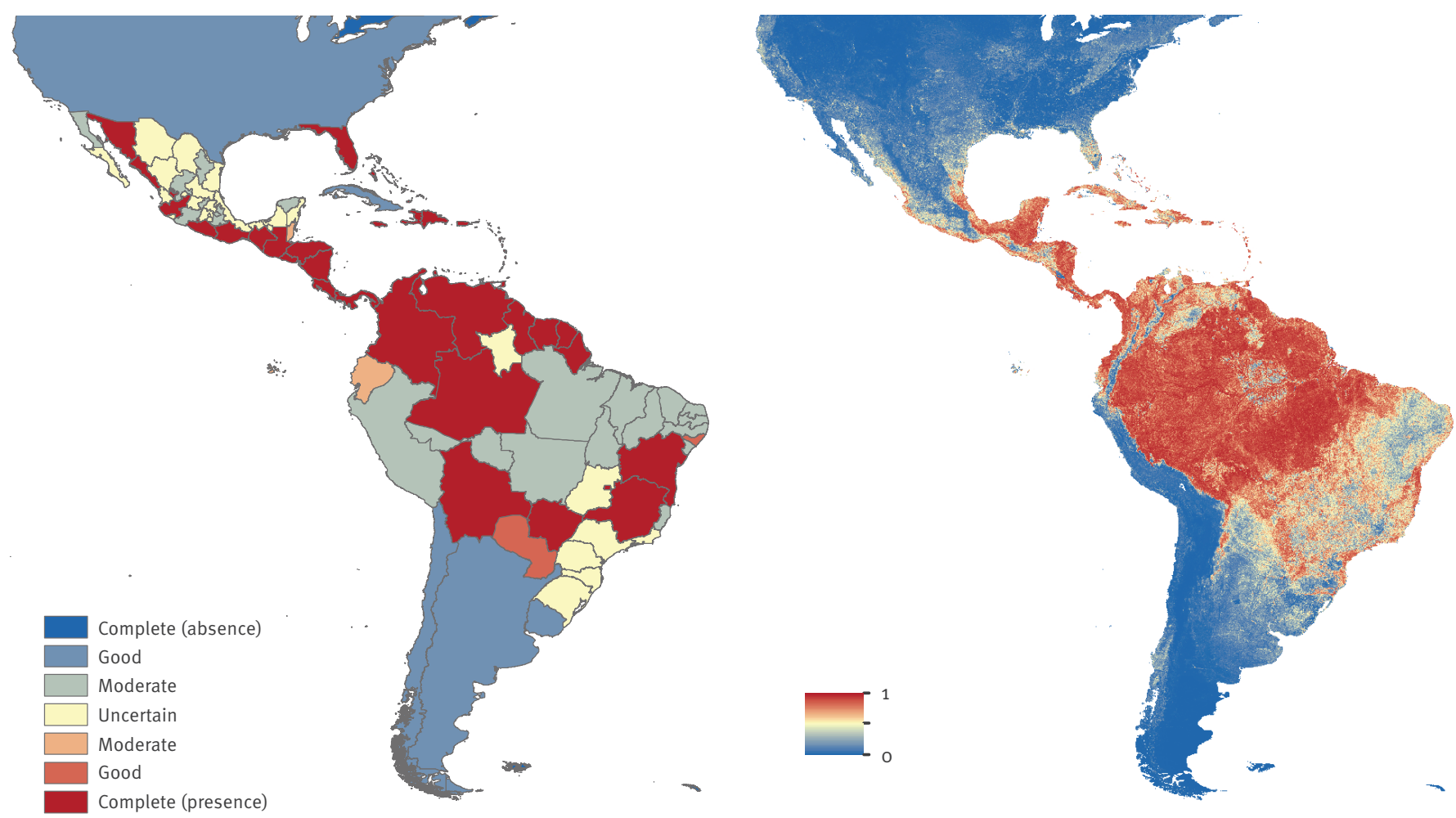

The colour legend for the evidence consensus ranges from red to blue representing complete consensus on presence to complete consensus on absence. Indeterminate consensus is in yellow. Evidence consensus is presented at Admin1 level for United States, Mexico, Argentina and Brazil, and Admino level for all other countries. The predicted environmental suitability map is presented in similar colours with 1 and $o$ representing most and least suitability for chikungunya virus, respectively.

\section{FIGURE 5}

Evidence consensus (a) and predicted environmental suitability (b) for chikungunya virus in Asia and Oceania, as of May 2015
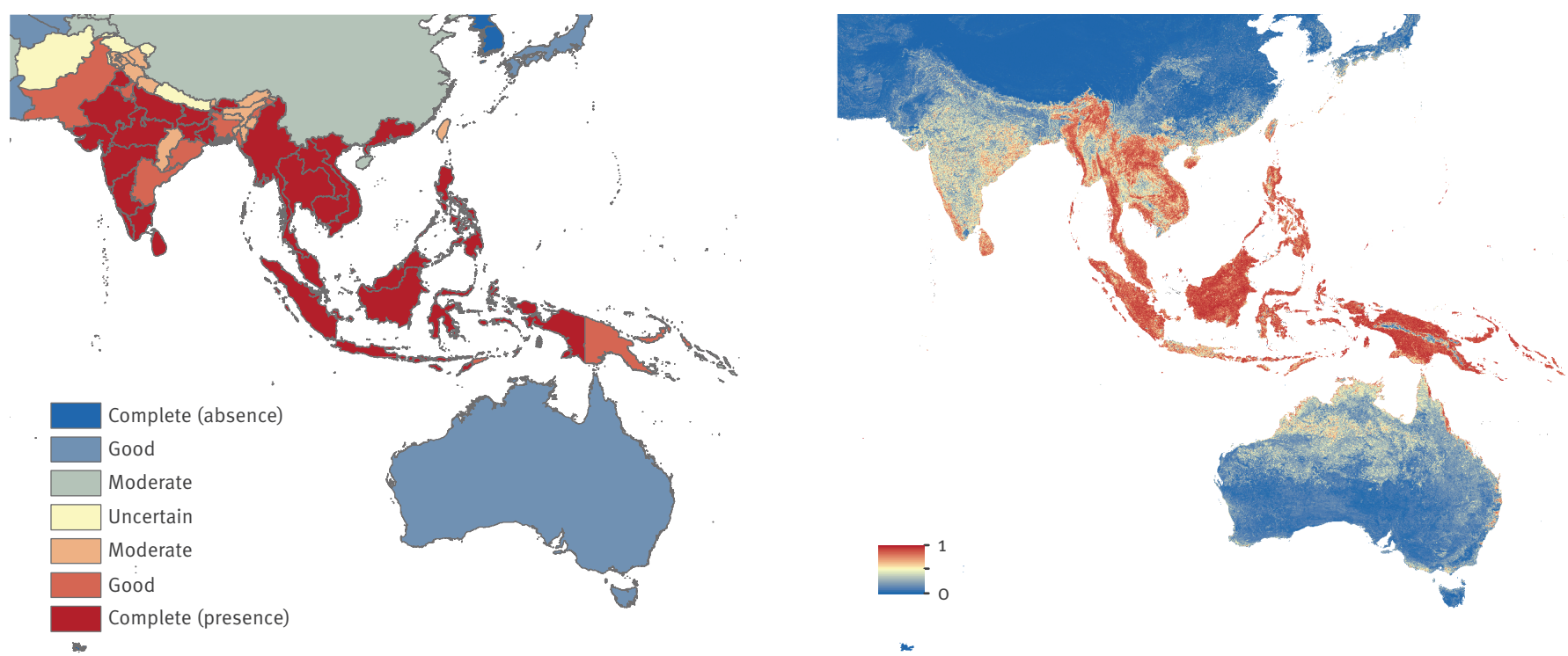

The colour legend for the evidence consensus ranges from red to blue representing complete consensus on presence to complete consensus on absence. Indeterminate consensus is in yellow. Evidence consensus is presented at Admin1 level for India and China, and country level for all other countries. The predicted environmental suitability map is presented in similar colours with 1 and o representing most and least suitability for chikungunya virus, respectively. 
TABLE 1

Summary of evidence used for chikungunya virus transmission consensus scoring

\section{Evidence category}

Health organisation status

Number of health organisations ${ }^{\mathrm{a}}$ reporting epidemics or local transmission of chikungunya virus

\begin{tabular}{|c|c|}
\hline 3 & $+6 /-6$ \\
\hline 2 & $+3 /-3$ \\
\hline \multicolumn{2}{|l|}{ Peer reviewed evidence } \\
\hline \multicolumn{2}{|l|}{ Date of chikungunya virus transmission occurrence } \\
\hline $2005-2015$ & 3 \\
\hline 1997-2004 & 2 \\
\hline Pre-1997 & 1 \\
\hline \multicolumn{2}{|l|}{ Diagnostic procedure } \\
\hline Polymerase chain reaction (PCR) & 3 \\
\hline IgM/IgG-based enzyme-linked immunosorbent assay (ELISA) and haemaglutination inhibition & 2 \\
\hline No specified test & 1 \\
\hline \multicolumn{2}{|l|}{ Combination score ${ }^{b}$} \\
\hline 3 types $^{b}$ & 3 \\
\hline 2 types $^{b}$ & 2 \\
\hline 1 types $^{b}$ & 1 \\
\hline \multicolumn{2}{|l|}{ Case data or health expenditure } \\
\hline \multicolumn{2}{|l|}{ Case data: most recent outbreak } \\
\hline $0-7$ years & 9 \\
\hline $7-14$ years & 6 \\
\hline $14-21$ years & 3 \\
\hline $28-35$ years & -3 \\
\hline$\geq 35$ years & -6 \\
\hline \multicolumn{2}{|l|}{ If no case data: health expenditure in 2014} \\
\hline HE «10o USD + sporadic cases & 6 \\
\hline HE «10o USD + sporadic cases & 3 \\
\hline 100 USD $\leq \mathrm{HE}<500$ USD & -3 \\
\hline $\mathrm{HE} \geq 500$ USD & -9 \\
\hline \multicolumn{2}{|l|}{ Supplementary evidence ${ }^{c}$} \\
\hline \multicolumn{2}{|l|}{ Number of evidence types ${ }^{c}$} \\
\hline 4 types $^{c}$ & 6 \\
\hline 3 types $^{c}$ & 4 \\
\hline 2 types $^{c}$ & 2 \\
\hline
\end{tabular}

a The three health organisations considered included (i) the United States Centers for Disease Control and Prevention (CDC), (ii) the Global Infectious Disease and Epidemiology Network (GIDEON) and (iii) the World Health Organization (WHO) and Pan American Health Organization (PAHO) taken together.

${ }^{b}$ The types of evidences include (i) reports from health organisations, (ii) the date of chikungunya virus transmission occurrence, and (iii) the diagnostic procedure.

' The types of evidences include (i) mosquito presence, (ii) dengue presence, (iii) travel advisories, and (iv) HealthMap.

were then cross-checked manually and for each occurrence point, the probability of occurrence for both vectors was extracted (Figure $1 \mathrm{c}$ and $\mathrm{d}$ ) and records deemed implausible (probability of occurrence for both vectors individually fell below 70\%) [17] were removed. The cut-off at $70 \%$ was chosen based on a qualitative interpretation of the Ae. aegypti and Ae. albopictus probability of occurrence maps, where $>90 \%$ of the geographical spaces with non-zero probabilities were recorded between vector presence $70 \%$ to $100 \%$.

\section{Evidence consensus}

The evidence consensus is a summary statistic representing confidence in the presence or absence of CHIKV for a given political region. We calculated evidence consensus scores at a national level for all countries apart from Argentina, Brazil, China, France, India, Italy, Mexico, and the US, where sufficient detailed epidemiological information was available to distinguish within-country epidemiological, environmental, and economic variability. Smaller countries were not considered for subnational assessment. 


\section{TABLE 2}

Relative contribution of each covariate in explaining the global environmental suitability of chikungunya virus and 95\% confidence interval, 1952-May 2015

\begin{tabular}{|l|c|}
\hline Variable & Mean (95\% confidence interval) \\
\hline Aedes aegypti & $46.16(43.45-48.74)$ \\
\hline EVI mean and range & $33.37(29.71-36.94)$ \\
\hline Urban accessibility & $7.42(6.52-8.50)$ \\
\hline Ae. albopictus & $6.70(5.78-7.60)$ \\
\hline LST mean and range & $5.98(4.83-6.90)$ \\
\hline Urbanisation & $0.30(0.15-0.70)$ \\
\hline
\end{tabular}

EVI: enhanced vegetation index; LST: land surface temperature.

Each evidence category was scored independently and category weights applied to reflect the level of detail each category provides: health organisation status (max. score 6), peer-reviewed evidence (max. 9), case data (max. 9) and supplementary evidence (max. 6). For example, if all three health organisations considered (i.e. PAHO/WHO, GIDEON and CDC) agreed on presence/absence of CHIKV, the country was assigned a score of $+6 /-6$. If two health organisations agreed on presence/absence, the country was assigned a score of $+3 /-3$. For each country, we identified peer-reviewed evidence to confirm CHIKV presence based on seroprevalence studies, polymerase chain reaction (PCR) confirmation, case reports and local outbreaks. Each published study was scored independently based on date of disease occurrence and the average was taken and added to a score of accuracy, which was assigned based on diagnostic procedure. The case data category was scored based on the most recent outbreak year and pre-defined inter-epidemic period intervals to account for herd immunity $[18,19]$. In the absence of case data, healthcare expenditure (HE) was used as a proxy to indicate confidence in a country's ability to detect and report CHIKV cases and to supplement this category [13]. In addition, countries for which HE data were unavailable were assigned low $\mathrm{HE}$, and overseas territories were assigned the same $\mathrm{HE}$ as their parent nations.

For areas with conflicting reports from the input sources and recent geographical expansion (e.g. Mexico and Brazil), database records were crosschecked against expert knowledge and recent national reports. Supplementary evidence was extracted from peer-reviewed literature on mosquito presence, dengue presence, travel advisories (http://www.nathnac. org/travel), and the HealthMap database (http://www. healthmap.org) and scored based on number of evidence types. The HealthMap database contains data extracted from online news sources and alerts from official public health sources (e.g. ministries of public health) and was available from 2006 to 2014 . We also used data on the global evidence consensus on dengue transmission from Brady et al. [13] to supplement data on the presence of other arboviruses for each country. See Table 1 for a summary and Brady et al. [13] for additional details on the scoring process.

The scores from all previously described evidence categories were summed, divided by the maximum potential score, 30 and multiplied by 100 . The evidence consensus was then mapped based on seven equidistant categories: complete evidence for presence ( $>71.42$ to $\leq 100$ ) or absence $(>-100$ to $\leq-71.42)$, good evidence for presence $(>42.84$ to $\leq 71.42)$ or absence $(>-71.42$ to $\leq-42.84]$, moderate evidence for presence ( $>14.27$ to $\leq 42.84)$ or absence $(>-42.84$ to $\leq-14.27)$, and indeterminate evidence $(>-14.27$ to $\leq 14.27)$.

\section{Environmental risk maps}

A boosted regression tree (BRT) modelling approach was applied to derive probabilistic global environmental risk maps for CHIKV. BRT models combine the strengths of regression trees with boosting and are frequently used in species distribution modelling (SDM) [20] with the overall aim to identify areas that exhibit similar conditions to areas where the disease has been reported. These statistical models heavily depend on the input occurrence database. We included the following globally available environmental and socioeconomic covariates in our models: (i) probabilistic global niche maps for Ae. aegypti and Ae. albopictus [17]; (ii) land surface temperature (LST); (iii) a categorical differentiation in urban, peri-urban and rural environments, as well as their accessibility; and (iv) enhanced vegetation index (EVI).

\section{Aedes aegypti and Aedes albopictus suitability}

We constructed probabilistic global Aedes risk maps based on the most comprehensive globally representative occurrence dataset for both Ae. aegypti and Ae. albopictus species and a set of contemporary environmental covariates explaining their global distribution (Figure $1 \mathrm{c}$ and d) [17,21].

\section{Land surface temperature (LST)}

Temperature is one of the most important determinants of Aedes survival [22]. We used MODIS daytime LST after processing through a gap-filling algorithm to account for variation in temperature globally [23] (Figure $1 \mathrm{~g}$ and h). Additionally, transmission of CHIKV is dependent on the persistent presence of the disease vectors, Ae. aegypti and Ae. albopictus mosquitoes. Both adult female longevity and length of first gonotrophic cycle are temperature dependent. We used the combined temperature suitability of population persistence for both species to identify areas where by proxy we expect the geographical limits of transmission of CHIKV to be located $[13,15]$. The binary outputs of this model were used as a mask to exclude the placement of 10,000 pseudo-absences inside their physiologically plausible range. 
Enhanced vegetation index (EVI)

Water availability is another important factor for CHIKV transmission, as it has been shown, for example, that mosquitoes may cluster around houses if containers with standing water due to precipitation are present [24]. EVI measures vegetation canopy greenness and can be used as a proxy for soil surface-level moisture that are associated with the presence of vector larval development sites [25]. We used range and mean values of MODIS EVI after processing through a gap-filling algorithm described in Weiss et al. [23] (Figure 1e and f).

\section{Urbanisation}

To account for differences in urban, peri-urban and rural environments we built a categorical variable by supplementing the projected 2010 Global Rural Urban Mapping Project (GRUMP) urban rural categories with land-cover classes using night-time light satellite imagery and population density based on the most up-to-date national censuses available at the smallest available administrative unit [26]. A gridded surface of $5 \mathrm{~km} \times 5 \mathrm{~km}$ cells was generated with each pixel representing urban, peri-urban, or rural areas (Figure $1 b$ ).

\section{Urban accessibility}

Human movement defines the spatial scale of transmission of most communicable diseases. To account for areas that are highly accessible, we used a friction surface that shows pixel level travel time through the respective region of interest [27] (Figure 1a).

We then applied an ensemble BRT using 180 submodels to derive probabilistic estimates and underlying uncertainty at a $5 \mathrm{~km} \times 5 \mathrm{~km}$ resolution. We also estimated populations-at-risk of CHIKV transmission. For each occurrence record we identified the maximum risk probability of transmission within a buffer of 10 $\mathrm{km}$ around the occurrence record to derive a threshold value of probability of occurrence that should be included in the population-at-risk estimate. We then converted the continuous surface of transmission risk into a binary at-risk/not-at-risk classification. Finally, we extracted the population living in areas of potential transmission using a global population surface.

\section{Results}

The data abstraction yielded 528 occurrence points from peer-reviewed evidence and health organisations. The distribution of occurrence points by region is shown in Figure 2. Individually, WHO (+ PAHO), CDC and GIDEON implicated 60, 96, and 70 countries with reported CHIKV transmission, respectively. However, based on evidence from all available sources, we identified 94 countries with good or better evidence consensus on CHIKV presence from which $47 \%$ were in Asia and Africa and $44 \%$ in the Americas. Of the 94 countries, at total of $24,41,20$, two and seven were located in Africa, the Americas, Asia, Europe and Oceania, respectively.
High probability of Ae. aegypti occurrence was the strongest predictor for environmental suitability with a mean contribution of $46 \%$ (Table 2 ). Based on our environmental suitability predictions, we estimate that 1.3 billion people are living in areas of potential CHIKV transmission.

Global consensus on CHIKV presence or absence based on the evidence consensus scoring system and predictions of environmental suitability for CHIKV are further presented in for Africa and Europe, the Americas, and Asia and Oceania, respectively. The evidence ranges from complete presence to complete absence.

\section{Africa and Europe}

Most of the African countries with reliable evidence on presence and absence were located in Central and North Africa, respectively. The strong evidence for CHIKV presence in Central Africa was aided by incountry seroprevalence studies such as in Cameroon and Gabon and confirmed imported cases to nonendemic regions $[28,29]$. There was insufficient data to determine presence or absence for some countries in Africa such as Ghana, Ethiopia, and Mozambique, due to a general lack of data and poor surveillance. South Africa, for example, had a reported outbreak in 1977 [30], but there have been no new reports of outbreaks or seroprevalence studies. Most countries in Central and parts of West Africa with strong evidence on CHIKV presence also demonstrated high environmental suitability for CHIKV (Figure 3b). We estimated that over 240 million people live in areas at risk for CHIKV transmission in Africa.

Autochthonous transmission of CHIKV in Europe has been reported in Ravenna, northern Italy in 2007 and in the south-eastern French city of Fréjus in 2010 and Montpellier, southern France in 2014 (Figure 3a). Prior to these cases, CHIKV in Europe was limited to travelrelated cases. Genomic micro-evolution of CHIKV in Reunion facilitated transmission by $A e$. albopictus [31] in strains belonging to the so called Indian Ocean lineage (IOL) which increased the likelihood of CHIKV outbreaks in Europe. We predicted moderate environmental suitability for parts of France, Spain, Germany and Italy (Figure $3 \mathrm{~b}$ ), which is in agreement with previous projections of increased risk of CHIKV for France, northern Italy and the Pannonian Basin [32].

\section{Americas}

Systematic reporting of confirmed cases to PAHO provided well-documented evidence for CHIKV occurrence in the Americas. As of 13 March 2015, 45 countries had reported local transmission of CHIKV, while the evidence consensus identified $90 \%$ of countries and island states with good or better consensus (Figure $4 \mathrm{a}$ ). Laboratory confirmation of autochthonous cases made a major contribution in defining the South and Central America's higher consensus cluster on presence. The evidence strength varied across states for Brazil and Mexico. Brazil had strong evidence on presence in 
south-central states; indeterminate evidence for several southern states and Amazonas state in the north; and moderate evidence in central and northern states. In contrast, the environmental suitability map predicted that coastal Brazil and areas in the Amazon were highly suitable for CHIKV transmission. Peru and states in Brazil where evidence currently suggest absence, demonstrated a moderate to high suitability for CHIKV (Figure 4b).

Our models identified Ae. aegypti as the strongest predictor of CHIKV suitability, which is the primary vector implicated in the outbreak in most of the Caribbean and the Americas [33,34]. Based on the environmental suitability predictions, we estimated that over 260 million people live in areas at risk for CHIKV transmission in the Americas.

\section{Asia and Oceania}

CHIKV presence is well documented for most countries and islands in south and south-east Asia. Persistent vector populations and naïve populations contributed to notable pre-2000 CHIKV outbreaks in India, Myanmar/Burma, Philippines, Sri Lanka, Thailand and Vietnam [35,36]. In the last decade, outbreaks have been reported in India, Indian Ocean islands, Indonesia, Sri Lanka, and Thailand, following a 41-year period of quiescence [37,38]. Good to better consensus on CHIKV presence was reported for $80 \%$ of Asian countries and clusters of complete consensus were observed ranging from Myanmar/Burma to Vietnam for south Asia and Indonesia to Papua New Guinea for south-east Asian islands (Figure 5a). Complete consensus on CHIKV presence was noted for Guangdong province, China, where an outbreak was reported in 2010 [39]. With the exception of Chhattisgarh in Central India and smaller states in the north-east and north-west, most Indian states had good consensus on CHIKV presence. There was insufficient data on presence or absence of CHIKV in Nepal. Despite reports of large outbreaks in neighbouring India and indication of possible local transmission, there are no published national seroprevalence surveys for Nepal and CHIKV cases are possibly misdiagnosed as typhoid or other bacterial or viral fevers [40].

The environmental suitability map strongly complemented the evidence consensus with high risk of CHIKV transmission predicted for most south-east Asia islands and parts of south-east Asia, and moderate risk predicted for India (Figure $5 \mathrm{~b}$ ). Predictions of suitability for CHIKV transmission in China were patchy, with high risk predicted for regions where local CHIKV transmission has been observed. High prevalence has been noted for other arboviral diseases in southeast coastal regions, and northern and inland regions $[41,42]$, signalling suitability and risk for CHIKV transmission. We estimated that over 270 million people live in areas at risk for CHIKV in Asia.

\section{Discussion}

We present the global distribution and predict environmental suitability for CHIKV transmission. In this analysis we combined extensive evidence available at multiple geographical scales and integrated them into a well-established disease mapping approach. We identified areas particularly in Africa where CHIKV status is uncertain (e.g. Ethiopia, Mozambique) although there is high environmental suitability, presence of disease vectors and reported outbreaks in neighbouring countries such as Tanzania. We also predicted high environmental suitability for some regions but observed moderate evidence for absence of local transmission, such as in the Amazon basin. The former could be attributable to inadequate surveillance or misdiagnosis, while the latter could be explained by the recent emergence of CHIKV and the endemicity of dengue in these regions. Our comprehensive assessment of these evidence gaps enables the identification of areas where surveillance, seroprevalence studies, and vector control measures are increasingly important [43].

Despite the growing economic and public health impact of CHIKV outbreaks, there have not been any thorough assessments of knowledge gaps or high-resolution distribution maps of the disease. This is the first comprehensive assessment of the current spatial extent and environmental suitability of CHIKV transmission globally. We considered extensive available evidence from 1952 when CHIKV was first identified in Tanzania. We observed the following: (i) CHIKV evidence is dynamic, re-emerging and due to its high attack rate likely to cause outbreaks with significant timelags; (ii) misclassification and delay in reporting may influence the perception of current vs. former presence; (iii) while there is strong evidence for some areas (e.g. Asia), data on CHIKV occurrence in many parts of Africa remain sparse.

Similar to dengue [44], CHIK appears to be an underrecognised problem in Africa. Our environmental suitability maps indicate moderate to strong risk for CHIKV transmission in most of West, Central and East African countries. However, there is insufficient data to determine presence or absence for several countries in West Africa and parts of East Africa. Seroprevalence surveys are needed for countries with indeterminate status.

Additionally, co-occurrence and comorbidity of CHIKV and other arboviruses have been reported, which stresses the importance for better diagnostic tests. Furthermore, since dengue and CHIKV share the same primary vectors, similar estimates for population-atrisk would be expected. Population at risk of dengue virus transmission has been estimated at 3.97 billion people [13], which is far more than the 1.3 billion predicted for CHIKV. However, the estimates for dengue were derived on a national level, while our estimates are at a $5 \mathrm{~km} \times 5 \mathrm{~km}$ spatial scale. 
Improvements, such as the identification of the primary vector for each occurrence record can be made as new data become available. Particularly in Africa and Micronesia it is hypothesised that other mosquito vectors (Ae. afticanus, Ae. luteocephalus, Ae. furcifertaylori and $A e$. hensilli) are responsible for transmitting CHIKV between humans [45]. Data on their geographical distribution however remains sparse and could not be integrated in the current analysis. Furthermore, since the maps are derived using long-term average covariate layers, they identify areas that are at risk of transmission at any time during the year but do not consider explicitly how seasonality or human mobility affects transmission; these factors influence the short and long-term dynamics of CHIKV transmission. In addition, there are a number of other factors that may influence the probability of infection that relate to social aspects, such as, housing quality, poverty, susceptibility of the population, and the use of air-conditioning. Such factors were not included in our study since no reliable data are available on a global scale.

Furthermore, there are some challenges in developing a global model to predict environmental suitability for CHIKV transmission. For instance, although there have been reports of major outbreaks of CHIKV in India, the environmental suitability models predicted moderate environmental suitability for CHIKV in these regions. The spatial variability observed for India is similar to the Ae. albopictus distribution map [17]. This suggests that our modelling approach strongly mirrors $A e$. albopictus distribution in India, which has been implicated in recent outbreaks, but fails to capture the distribution of other covariates. Similarly, in Europe where there have been documented outbreaks of CHIKV in France, and Italy, risk appears to be low in our prediction. Modelling the likely distribution of CHIKV based on Ae. albopictus which is well distributed in southern Europe could help refine our approach. However, as transmission of CHIKV is relatively low in Europe, our results indicate the likely lower relative global contribution of Ae. albopictus compared with Ae. aegypti. Additionally, our maps indicate high transmission risk in small island states that have experienced large outbreaks in the last decade. However, herd immunity was not incorporated into the environmental risk models, implying that small countries or islands with (>50\% attack rates) might have a lower risk of CHIKV outbreaks despite environmental suitability. These observations highlight the need for region or countryspecific modelling, which would be most beneficial for local public health policy decisions. These maps can however be used as baseline layers for future projections of CHIKV and compared with global maps of dengue [12].

Despite these limitations, the usefulness of these maps cannot be understated, especially given the CHIKV's currently expanding range in the Americas and elsewhere. By synchronising the evidence consensus, environmental suitability prediction maps and population at-risk estimates, areas most in need of CHIKV surveillance resources were identified. It is equally important that these maps are updated as soon as new information becomes available, such as recent reports of local CHIKV transmission in states of Brazil, and Peru and this will be achieved by incorporating the results of this study into the Atlas of Baseline Risk Assessment for Infectious Diseases (ABRAID, at www.abraid.ox.ac.uk).

\section{Acknowledgements}

We thank Maria Devine for proofreading.

EON is supported by funding from the National Institute of Environmental Health Sciences of the National Institutes of Health (\#Ko1ES025438). MUGK receives funding from the International research Consortium on Dengue Risk Assessment Management and Surveillance (IDAMS; European Commission 7th Framework Programme (21893)). NG is supported by a University of Melbourne McKenzie fellowship. DMP is funded by a Sir Richard Southwood Graduate Scholarship from the Department of Zoology at the University of Oxford. OJB is funded by a grant from the Bill \& Melinda Gates Foundation (\#OPP1053338). CLM is funded by a grant from the Bill \& Melinda Gates Foundation (\#OPP1093011). MAJ is supported by the Models of Infectious Disease Agent Study program (cooperative agreement \#1 U54GMo88558). PWG is a Career Development Fellow (\#Ko0669X) jointly funded by the UK Medical Research Council (MRC) and the UK Department for International Development (DfID) under the MRC/DfID Concordat agreement and receives support from the Bill \& Melinda Gates Foundation (\#OPP1068048, \#OPP1106023). KK is funded by the Canadian Institutes for Health Research. SIH received a grant from the Research for Health in Humanitarian Crises ( $22 \mathrm{HC}$ ) Programme, managed by ELRHA (\#13468) which also supported NG \& MUGK. The Research for Health in Humanitarian Crises ( $22 \mathrm{HC}$ ) programme aims to improve health outcomes by strengthening the evidence base for public health interventions in humanitarian crises. Visit www.elrha.org/work/r2hc for more information. The $f 8$ million $22 \mathrm{HC}$ programme is funded equally by the Wellcome Trust and DFID, with Enhancing Learning and Research for Humanitarian Assistance (ELRHA) overseeing the programme's execution and management. $\mathrm{SIH}$ is funded by a Senior Research Fellowship from the Wellcome Trust (\#095066), and grants from the Bill \& Melinda Gates Foundation (OPP1119467, OPP1093011, OPP1106023 and OPP1132415). JSB acknowledges funding from NIH National Library of Medicine (\#R01LM010812-05) and from the Bill \& Melinda Gates Foundation (\#OPP1093011). The funders had no role in study design, data collection and interpretation, or the decision to submit the work for publication.

\section{Conflict of interest}

None declared.

\section{Authors' contributions}

Elaine Nsoesie and Moritz Kraemer performed data collection and analysis, and drafted the manuscript. Catherine Moyes reviewed data for accuracy. Michael Johansson served as the expert reviewer. Nick Golding, Oliver Brady and David Pigott advised on methods. John Brownstein and Simon Hay provided resources. All authors contributed to the writing and editing of the manuscript. 


\section{References}

1. Weaver SC, Lecuit M. Chikungunya virus and the global spread of a mosquito-borne disease.N Engl J Med. 2015;372(13):12319. DOI: 10.1056/NEJMra1406035 PMID: 25806915

2. Ross RW. The Newala epidemic. III. The virus: isolation, pathogenic properties and relationship to the epidemic.J Hyg (Lond). 1956;54(2):177-91. DOI: 10.1017/So022172400044442 PMID: 13346078

3. Powers $\mathrm{AM}$, Logue $\mathrm{CH}$. Changing patterns of chikungunya virus: re-emergence of a zoonotic arbovirus.J Gen Virol. 2007;88(Pt 9):2363-77. DOI: 10.1099/vir.0.82858-o PMID: 17698645

4. Weaver SC. Arrival of chikungunya virus in the new world: prospects for spread and impact on public health.PLoS Negl Trop Dis. 2014;8(6):e2921. DOI: 10.1371/journal.pntd.0002921 PMID: 24967777

5. Volk SM, Chen R, Tsetsarkin KA, Adams AP, Garcia TI, Sall AA, et al. Genome-scale phylogenetic analyses of chikungunya virus reveal independent emergences of recent epidemics and various evolutionary rates. J Virol. 2010;84(13):6497-504. DOI: 10.1128/JVI.01603-09 PMID: 20410280

6. Staples JE, Breiman RF, Powers AM. Chikungunya fever: an epidemiological review of a re-emerging infectious disease. Clin Infect Dis. 2009;49(6):942-8. DOI: 10.1086/605496 PMID: 19663604

7. Chahar HS, Bharaj P, Dar L, Guleria R, Kabra SK, Broor S. Coinfections with chikungunya virus and dengue virus in Delhi, India.Emerg Infect Dis. 2009;15(7):1077-80. DOI: 10.3201/ eid1507.080638 PMID: 19624923

8. Weaver SC. Arrival of chikungunya virus in the new world: prospects for spread and impact on public health.PLoS Negl Trop Dis. 2014;8(6):e2921. DOI: 10.1371/journal.pntd.0002921 PMID: 24967777

9. Chang LJ, Dowd KA, Mendoza FH, Saunders JG, Sitar S, Plummer SH, et al., VRC 311 Study Team. Safety and tolerability of chikungunya virus-like particle vaccine in healthy adults: a phase 1 dose-escalation trial.Lancet. 2014;384(9959):2046-52. DOI: 10.1016/S0140-6736(14)61185-5 PMID: 25132507

10. Johansson MA. Chikungunya on the move.Trends Parasitol. 2015;31(2):43-5. DOI: 10.1016/j.pt.2014.12.008 PMID: 25649340

11. Kraemer MUG, Hay SI, Pigott DM, Smith DL, Wint GRW, Golding N. Progress and Challenges in Infectious Disease Cartography.Trends Parasitol. 2016;32(1):19-29. DOI: 10.1016/j. pt.2015.09.006 PMID: 26604163

12. Bhatt S, Gething PW, Brady OJ, Messina JP, Farlow AW, Moyes $\mathrm{CL}$, et al. The global distribution and burden of dengue. Nature. 2013;496(7446):504-7. DOI: 10.1038/nature12060 PMID: 23563266

13. Brady OJ, Gething PW, Bhatt S, Messina JP, Brownstein JS, Hoen AG, et al. Refining the global spatial limits of dengue virus transmission by evidence-based consensus. PLoS Negl Trop Dis. 2012;6(8):e176o. DOI: http://dx.doi.org/10.1371/ journal.pntd.0001760

14. Messina JP, Kraemer MU, Brady OJ, Pigott DM, Shearer FM, Weiss DJ, et al. Mapping global environmental suitability for Zika virus. eLife. 2016;5:e15272. DOI: 10.7554/eLife.15272 PMID: 27090089

15. Pigott DM, Bhatt S, Golding N, Duda KA, Battle KE, Brady OJ, et al. Global distribution maps of the leishmaniases. eLife. 2014;3. DOI: 10.7554/eLife.02851 PMID: 24972829

16. Messina JP, Brady OJ, Pigott DM, Brownstein JS, Hoen AG, Hay Sl. A global compendium of human dengue virus occurrence. Sci Data. 2014;1:140004. DOI: 10.1038/sdata.2014.4 PMID: 25977762

17. Kraemer MU, Sinka ME, Duda KA, Mylne AQ, Sheare FM, Barker CM, et al. The global distribution of the arbovirus vectors Aedes aegypti and Ae. albopictus. eLife. 2015;4:e08347. DOI: 10.7554/eLife.08347 PMID: 26126267

18. Gérardin P, Guernier V, Perrau J, Fianu A, Le Roux K, Grivard P, et al. Estimating Chikungunya prevalence in La Réunion Island outbreak by serosurveys: two methods for two critical times of the epidemic. BMC Infect Dis. 2008;8(1):99. DOI: 10.1186/14712334-8-99 PMID: 18662384

19. Nitatpattana N, Kanjanopas K, Yoksan S, Satimai W, Vongba N, Langdatsuwan S, et al. Long-term persistence of Chikungunya virus neutralizing antibodies in human populations of North Eastern Thailand. Virol J. 2014;11(1):183. DOI: 10.1186/1743422X-11-183 PMID: 25330992

20. Phillips SJ, Dudík M, Elith J, Graham CH, Lehmann A, Leathwick J, et al. Sample selection bias and presence-only distribution models: implications for background and pseudo-absence data. Ecol Appl. 2009;19(1):181-97. DOI: 10.1890/07-2153.1 PMID: 19323182
21. Kraemer MU, Sinka ME, Duda KA, Mylne A, Shearer FM, Brady OJ, et al. The global compendium of Aedes aegypti and Ae. albopictus occurrence. Sci Data. 2015;2:150035. DOI: 10.1038/ sdata.2015.35 PMID: 26175912

22. Brady OJ, Golding N, Pigott DM, Kraemer MU, Messina JP, Reiner RC, et al. Global temperature constraints on Aedes aegypti and Ae. albopictus persistence and competence for dengue virus transmission. Parasit Vectors. 2014;7(1):338. DOI: 10.1186/1756-3305-7-338 PMID: 25052008

23. Weiss DJ, Atkinson PM, Bhatt S, Mappin B, Hay SI, Gething PW. An effective approach for gap-filling continental scale remotely sensed time-series.ISPRS I Photogramm Remote Sens. 2014;98:106-18. DOI: 10.1016/j.isprsjprs.2014.10.001 PMID: 25642100

24. Luz C, Tai MHH, Santos AH, Silva HHG. Impact of moisture on survival of Aedes aegypti eggs and ovicidal activity of Metarhizium anisopliae under laboratory conditions. Mem Inst Oswaldo Cruz. 2008;103(2):214-5. DOI: 10.1590/So07402762008000200016 PMID: 18425277

25. Estallo EL, Lamfri MA, Scavuzzo CM, Almeida FF, Introin MV, Zaidenberg $M$, et al. Models for predicting Aedes aegypti larval indices based on satellite images and climatic variables. J Am Mosq Control Assoc. 2008;24(3):368-76. DOI: 10.2987/5705.1 PMID: 18939688

26. Balk DL, Deichmann U, Yetman G, Pozzi F, Hay SI, Nelson A. Determining global population distribution: methods, applications and data.Adv Parasitol. 2006;62:119-56. DOI: 10.1016/So065-308X(05)62004-0 PMID: 16647969

27. Uchida H, Nelson A. Agglomeration index: Towards a New Measure of Urban Concentration. Background paper for the World Bank's World Development Report. Washington D.C.: World Bank; 2009.

28. Demanou M, Antonio-Nkondjio C, Ngapana E, Rousset D, Paupy C, Manuguerra J-C, et al. Chikungunya outbreak in a rural area of Western Cameroon in 2006: A retrospective serological and entomological survey. BMC Res Notes. 2010;3(1):128. DOI: 10.1186/1756-0500-3-128 PMID: 20444282

29. Peyrefitte CN, Bessaud M, Pastorino BAM, Gravier P, Plumet $\mathrm{S}$, Merle OL, et al. Circulation of Chikungunya virus in Gabon, 2006-2007. J Med Virol. 2008;80(3):430-3. DOI: 10.1002/ jmv.21090 PMID: 18205212

30. Sudeep AB, Parashar D. Chikungunya: an overview.J Biosci. 2008;33(4):443-9. DOI: 10.1007/S12038-008-0063-2 PMID: 19208970

31. Schuffenecker I, Iteman I, Michault A, Murri S, Frangeul L, Vaney M-C, et al. Genome microevolution of chikungunya viruses causing the Indian Ocean outbreak. PLoS Med. 2006;3(7):e263. DOI: 10.1371/journal.pmed.0030263 PMID: 16700631

32. Fischer D, Thomas SM, Suk JE, Sudre B, Hess A, Tjaden NB, et al. Climate change effects on Chikungunya transmission in Europe: geospatial analysis of vector's climatic suitability and virus' temperature requirements. Int J Health Geogr. 2013;12(1):51. DOI: 10.1186/1476-072X-12-51 PMID: 24219507

33. Leparc-Goffart I, Nougairede A, Cassadou S, Prat C, de Lamballerie X. Chikungunya in the Americas. Lancet. 2014;383(9916):514. DOI: 10.1016/S0140-6736(14)60185-9 PMID: 24506907

34. Van Bortel W, Dorleans F, Rosine I, Blateau A, Rousset D, Matheus S, et al. Chikungunya outbreak in the Caribbean region, December 2013 to March 2014, and the significance for Europe. Euro Surveill. 2014;19(13):20759. DOI: 10.2807/15607917.ES2014.19.13.20759 PMID: 24721539

35. Pulmanausahakul R, Roytrakul S, Auewarakul P, Smith DR. Chikungunya in Southeast Asia: understanding the emergence and finding solutions. Int J Infect Dis. 2011;15(10):e671-6. DOI: 10.1016/j.ijid.2011.06.002 PMID: 21775183

36. Lanciotti RS, Valadere AM, . Transcontinental movement of Asian genotype chikungunya virus. Emerg Infect Dis. 2014;20(8):1400-2. DOI: 10.3201/eid2008.140268 PMID: 25076384

37. Azami NAM, Salleh SA, Shah SA, Neoh HM, Othman Z, Zakaria $S Z S$, et al. Emergence of chikungunya seropositivity in healthy Malaysian adults residing in outbreak-free locations: chikungunya seroprevalence results from the Malaysian Cohort. BMC Infect Dis. 2013;13(1):67. DOI: 10.1186/1471-233413-67 PMID: 23379541

38. Pulmanausahakul R, Roytrakul S, Auewarakul P, Smith DR. Chikungunya in Southeast Asia: understanding the emergence and finding solutions. Int J Infect Dis. 2011;15(10):e671-6. DOI: 10.1016/j.ijid.2011.06.002 PMID: 21775183

39. Wu D, Wu J, Zhang Q, Zhong H, Ke C, Deng X, et al. Chikungunya outbreak in Guangdong Province, China, 2010. Emerg Infect Dis. 2012;18(3):493-5. DOI: 10.3201/ eid1803.110034 PMID: 22377135 
40. Pun SB, Bastola A, Shah R. First report of Chikungunya virus infection in Nepal.J Infect Dev Ctries. 2014;8(6):790-2. DOI: 10.3855/jidc.3701 PMID: 24916880

41. Wang J, Zhang H, Sun X, Fu S, Wang H, Feng Y, et al.

Distribution of mosquitoes and mosquito-borne arboviruses in Yunnan Province near the China-Myanmar-Laos border. Am J Trop Med Hyg. 2011;84(5):738-46. DOI: 10.4269/ ajtmh.2011.10-0294 PMID: 21540383

42. Wu J-Y, Lun Z-R, James AA, Chen X-G. Dengue Fever in mainland China.Am J Trop Med Hyg. 2010;83(3):664-71. DOI: 10.4269 / ajtmh.2010.09-0755 PMID: 20810836

43. Nunes MRT, Faria NR, de Vasconcelos JM, Golding N, Kraemer MU, de Oliveira LF, et al. Emergence and potential for spread of Chikungunya virus in Brazil. BMC Med. 2015;13(102):102. DOI: 10.1186/S12916-015-0348-x PMID: 25976325

44. Jaenisch T, Junghanss T, Wills B, Brady OJ, Eckerle I, Farlow A, et al. , Dengue in Africa Study Group. Dengue expansion in Africa-not recognized or not happening?Emerg Infect Dis. 2014;20(10):e140487. DOI: 10.3201/eid2010.140487 PMID: 25271370

45. Ledermann JP, Guillaumot L, Yug L, Saweyog SC, Tided M, Machieng $P$, et al. Aedes hensilli as a potential vecto of Chikungunya and Zika viruses. PLoS Negl Trop Dis. 2014;8(10):e3188. DOI: 10.1371/journal.pntd.0003188 PMID: 25299181

\section{License and copyright}

This is an open-access article distributed under the terms of the Creative Commons Attribution (CC BY 4.0) Licence. You may share and adapt the material, but must give appropriate credit to the source, provide a link to the licence, and indicate if changes were made.

This article is copyright of the authors, 2016. 